\title{
Aprendizagem Móvel na Produção Científica Indexada ao Scopus nos Anos de 2016 e 2017
}

\author{
Ernane Rosa Martins , Luís Manuel Borges Gouveia² \\ ${ }^{1}$ Instituto Federal de Goiás (IFG) \\ Caixa Postal 72.811-580 - Luziânia - GO - Brasil \\ ${ }^{2}$ Universidade Fernando Pessoa (UFP) \\ Caixa Postal 4249-004 - Porto - Portugal \\ ernane.martins@ifg.edu.br, lmbgeufp.edu.pt
}

\begin{abstract}
The objective of this work is to know the studies on mobile learning conducted in the last years indexed to Scopus database. To achieve this objective, the systematic literature review method sought to draw an overview of the scientific production related to this theme in 2016 and 2017, in order to allow the visualization of possible gaps to be deepened, enabling new research opportunities. As results are presented the characteristics of the selected publications within the given scope.
\end{abstract}

Resumo. O objetivo deste trabalho é conhecer os estudos sobre aprendizagem móvel realizados nos últimos anos indexados a base de dados Scopus. Para alcançar este objetivo buscou-se, através do método da revisão sistemática da literatura, traçar um panorama sobre a produção cientifica relacionada a este tema nos anos de 2016 e 2017, com a finalidade de permitir a visualização de possiveis lacunas a serem aprofundadas, possibilitando novas oportunidades de pesquisa. Como resultados são apresentadas as características das publicações selecionadas dentro do escopo determinado.

\section{Introdução}

A aprendizagem móvel (Mobile Learning) é um campo de pesquisa emergente e em expansão, devido à crescente mobilidade da sociedade atual (Martins et al., 2018). Os dispositivos móveis estão sendo usados para fornecer novos recursos aos alunos, tais como: aprendizado contextualizado e personalizado (Crompton, 2013). Atualmente tem ocorrido um aumento visível em investigar a amplitude, o proposito e a extensão da aprendizagem móvel na educação (Ally \& Prieto-Blázquez, 2014). O avanço acelerado nas novas funcionalidades dos dispositivos móveis, mostra que é fundamental manter uma coleção atualizada e sintetizada das pesquisas existentes, para possibilitar que a comunidade acadêmica mantenha-se atualizada na compreensão da aprendizagem móvel. Além disso, os pesquisadores necessitam continuar investigando questões não examinadas sobre a aprendizagem móvel (Foti, 2014). O objetivo deste trabalho é conhecer os estudos sobre aprendizagem móvel nos últimos anos. Para alcançar este objetivo buscou-se, através da revisão sistemática da literatura, traçar um panorama sobre a produção científica sobre o tema nos anos de 2016 e 2017, na base de dados SCOPUS, com a finalidade de visualizar possíveis lacunas e oportunidades de pesquisa. 


\section{Aprendizagem móvel}

A definição de aprendizagem móvel que frequentemente é utilizada pela comunidade acadêmica é "aprendizagem em múltiplos contextos, através de interações sociais e de conteúdo, usando dispositivos eletrônicos pessoais" (Crompton, 2013, p. 4.). Ao longo dos anos, vem sendo realizadas diversas revisões sobre aprendizagem móvel, cada qual contribui com informações importantes para que os pesquisadores entendam melhor a utilização dos dispositivos móveis nos ambientes de aprendizagem. Frohberg, Göth \& Schwabe (2009) realizaram uma revisão sobre aprendizagem móvel de 2002 a 2007. Descobrindo que a aprendizagem móvel pode fornecer melhor suporte para $\mathrm{o}$ aprendizado quando utilizada em contexto especifico e que a aprendizagem móvel provoca reflexão, comunicação e cooperação por parte dos alunos.

Hwang \& Tsai (2011) revisaram artigos de ensino móvel de 2001 a 2010, focando em características, como assuntos, séries e países de estudos. Wu et al. (2012) realizaram revisões de artigos de ensino móvel no fundamental e médio de 2003 a 2010, investigando várias características, como propósitos de pesquisa, métodos e resultados. Liu et al. (2014) revisaram os artigos de aprendizagem móvel de 2007 a 2012, investigando o design da pesquisa, os resultados de aprendizagem e o conteúdo acadêmico. Crompton et al. (2016) realizaram revisões dos estudos de aprendizagem móvel relacionados à ciência, de estudos a partir do ano 2000 em diante, revisando as características gerais, incluindo nível de série, metodologias e propósito. Sung, Chang \& Liu (2016) realizaram uma revisão para entender os efeitos da integração de dispositivos móveis no desempenho de aprendizado dos alunos de 1993 a 2013. Os estudos anteriores forneceram uma valiosa síntese das pesquisas sobre aprendizado móvel. Do mesmo modo, está presente revisão vem fornecer uma síntese atual da pesquisa móvel de aprendizagem nos anos de 2016 e 2017.

\section{Método e procedimentos da pesquisa}

Este estudo consiste em uma revisão sistemática da literatura científica sobre aprendizagem móvel. A revisão sistemática é realizada de forma a identificar, selecionar e sintetizar estudos primários de pesquisa para fornecer uma visão abrangente e confiável do tópico em estudo, para responder a uma pergunta específica (Gough et al., 2012). A revisão sistemática foi escolhida como método, por diversas características específicas, tais como: pode ser reprodutível, pode ser rapidamente atualizada, possibilita identificar lacunas no campo de pesquisa e fornece base confiável para tomada de decisão. As revisões sistemáticas são investigações científicas, com métodos pré-planejados, baseados na revisão de artigos provenientes de estudos originais disponíveis. Para Transfield, Denyer \& Smart (2003) a realização de uma revisão sistemática da literatura auxilia o pesquisador na compreensão de determinados fenômenos, por fornecer evidências sobre as práticas do estudo. A estratégia utilizada neste trabalho, teve como procedimentos quatro passos distintos, sendo eles:

$1^{\circ}$ Passo: Escolha da base de dados, optou-se pela base de dados SCOPUS, para poder conhecer como os estudos sobre aprendizagem móvel estão sendo apresentados de forma macro. A base de dados SCOPUS possui características específicas relevantes, tais como: é a maior base de dados de resumos e citações de literatura científica revisada por pares; mais de 22.000 títulos de periódicos de mais de 5.000 editoras internacionais; 
multidisciplinar, abrangendo diversas áreas. $2^{\circ}$ Passo: Escolha da palavra-chave e período de pesquisa, considerou-se realizar a busca nos últimos anos, de forma a analisar as publicações mais recentes sobre o tema, tendo o ano de 2017 como último ano de pesquisa, em virtude de muitos periódicos ainda não disponibilizarem suas literaturas completas sobre o tema no ano de 2018. Assim, a busca na base de dados abrangeu os anos de 2016 e 2017. Para palavra-chave, utilizou-se o termo "Mobile Learning", por ser amplamente conhecido. A busca ocorreu em: títulos, resumos e palavras-chave, limitando-se apenas à busca de artigos.

$3^{\circ}$ Passo: análise dos periódicos, realizou-se a leitura do título e do abstract de cada uma das 88 publicações selecionadas. Os artigos foram estudados em profundidade, sendo utilizados como critérios: objetivo da pesquisa; tipo de pesquisa; nível de análise; método de pesquisa; contexto da pesquisa; resultados encontrados com a aprendizagem móvel; recomendações para pesquisas futuras. $4^{\circ}$ Passo: Compreensão dos periódicos, buscou-se neste passo conhecer quais periódicos, campo de estudo e autores que mais publicaram dentro do escopo selecionado. O intuito principal desta parte foi aprofundar sobre o universo dos estudos sobre aprendizagem móvel.

\section{Análises e discussões das publicações selecionadas}

Verificou-se a partir da análise das publicações que há uma presença marcante da temática da aprendizagem móvel em alguns periódicos internacionais, como no caso do periódico International Journal of Interactive MobileTechnologies, seguida da Computers in Human Behavior e Computers \& Education. Conforme a Tabela 1.

Tabela 1. Número de artigos por periódicos.

\begin{tabular}{cc}
\hline Revistas e Periódicos & Número de artigos \\
\hline Computers in Human Behavior & 9 \\
\hline Computers \& Education & 7 \\
\hline Educational Technology \& Society & 6 \\
\hline International Review of Research in Open and Distributed Learning & 5 \\
\hline Interactive Technology and Smart Education & 5 \\
\hline Educational Technology Research and Development & 4 \\
\hline International Journal of Emerging Technologies in Learning & 4 \\
\hline Education and Information Technologies & 4 \\
\hline Journal of Research in Education Sciences & 4 \\
\hline South African Journal of Education & 2 \\
\hline Journal of Computing in Higher Education & 2 \\
\hline Journal of Educational Computing Research & 2 \\
\hline Journal of Computer Assisted Learning & 2 \\
\hline TechTrends & 2 \\
\hline Journal of Theoretical and Applied Information Technology & 2 \\
\hline Universal Access in the Information Society & 2 \\
\hline Journal of Educational Psychology & 2 \\
\hline Journal of Science Education and Technology & 1 \\
\hline Journal of Engineering Science and Technology Review & 1 \\
\hline
\end{tabular}




\begin{tabular}{|c|c|}
\hline Electronic Library & 1 \\
\hline ZDM: The International Journal on Mathematics Education & 1 \\
\hline IEEE & 1 \\
\hline Journal of Educators Online & 1 \\
\hline Multimedia Tools and Applications & 1 \\
\hline Journal of Applied Research in Higher Education & 1 \\
\hline Technology in Society & 1 \\
\hline Innovations in Education and Teaching International & 1 \\
\hline Journal Of Baltic Science Education & 1 \\
\hline Information Development & 1 \\
\hline Development and Learning in Organizations & 1 \\
\hline Research in Science Education & 1 \\
\hline Computer Standards \& Interfaces & 1 \\
\hline Quality and Quantity & 1 \\
\hline International Journal of Distance Education Technologies & 1 \\
\hline Computer Applications in Engineering Education & 1 \\
\hline Science of Computer Programming & 1 \\
\hline The Internet and Higher Education & 1 \\
\hline Transinformacao & 1 \\
\hline Language Learning \& Technology & 1 \\
\hline Information and Learning Science & 1 \\
\hline
\end{tabular}

Em outra análise feita com base nos dados coletados sobre o ranking dos autores que mais publicaram sobre o tema aprendizagem móvel nos anos de 2016 e 2017. Destaque para os autores com duas publicações. Conforme ilustrado na Tabela 2.

Tabela 2. Autores que mais publicaram.

\begin{tabular}{cc}
\hline $\begin{array}{c}\text { Crompton, Helen; Burke, Diane; Gregory, } \\
\text { Kristen. }\end{array}$ & $\begin{array}{c}\text { Computers \& Education, July 2017, Vol.110, } \\
\text { pp.51-63 }\end{array}$ \\
\hline $\begin{array}{c}\text { Crompton, Helen; Burke, Diane; Gregory, } \\
\text { Kristen. }\end{array}$ & $\begin{array}{c}\text { Journal of Science Education and Technology, } \\
\text { 2016, Vol.25(2), p.149-160 }\end{array}$ \\
\hline Sarrab, Mohamed; & $\begin{array}{c}\text { Computers in Human Behavior, February 2016, } \\
\text { Vol.55, pp.100-112 }\end{array}$ \\
\hline Sarrab, Mohamed; & $\begin{array}{c}\text { International Review of Research in Open and } \\
\text { Distributed Learning, 2016, Vol.17(4), p.331-349 }\end{array}$ \\
\hline Kokkalia, G. K; Drigas, A.S. & $\begin{array}{c}\text { International Journal of Interactive } \\
\text { MobileTechnologies, 2016, Vol.10(1), pp.60-67 }\end{array}$ \\
\hline Kokkalia, G. K; Drigas, A.S. & $\begin{array}{r}\text { International Journal of } \\
\text { Interactive MobileTechnologies, 2016, Vol.10(4), } \\
\text { pp.57-64 }\end{array}$ \\
\hline Burden, Kevin John; Kearney, Matthew & $\begin{array}{r}\text { Interactive Technology and Smart Education, } \\
\text { 2017, Vol.14(2), p.110-125 }\end{array}$ \\
\hline Burden, Kevin John; Kearney, Matthew & $\begin{array}{r}\text { Research in Science Education, 2016, Vol.46(2), } \\
\text { p.287-308 }\end{array}$ \\
\hline
\end{tabular}


Gunasegaran, T.; Azizan, S.N.

International Journal of

Interactive MobileTechnologies, 2016, Vol.10(3), pp.17-23

Gunasegaran, T.; Azizan, S.N.

International Journal of

Interactive MobileTechnologies, 2017, Vol.11(1), pp.116-132

\begin{tabular}{cc}
\hline Shih, Ju - Ling & $\begin{array}{c}\text { International Journal of Distance Education } \\
\text { Technologies, 2016, Vol.14(3), p.91-106 }\end{array}$ \\
\hline Shih, Ju - Ling & $\begin{array}{c}\text { Universal Access in the Information Society, } \\
\text { 2017, Vol.16(3), pp.653-665 }\end{array}$ \\
\hline $\begin{array}{c}\text { Zhang, Bingxue; Yin, Chuantao; David, } \\
\text { Bertrand; Xiong, Zhang }\end{array}$ & $\begin{array}{c}\text { Computer Applications in Engineering Education, } \\
\text { March 2016, Vol.24(2), pp.263-276 }\end{array}$ \\
\hline $\begin{array}{c}\text { Zhang, Bingxue; Yin, Chuantao; David, } \\
\text { Bertrand; Xiong, Zhang }\end{array}$ & $\begin{array}{c}\text { Science of Computer Programming, 1 November } \\
\text { 2016, Vol.129, pp.3-19 }\end{array}$ \\
\hline
\end{tabular}

Outra análise do presente trabalho diz respeito ao tipo de estudo utilizado pelos autores pesquisados. A maioria dos autores não identificaram se a pesquisa realizada foi qualitativa ou quantitativa. Mas em relação aos trabalhos que identificaram, a maioria utilizou método de pesquisa qualitativa. Conforme apresentado na Figura 1.

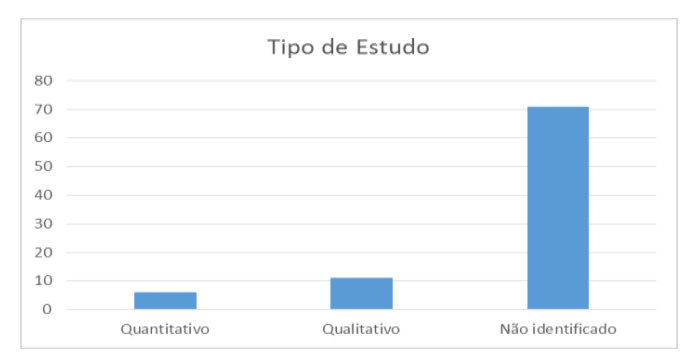

Figura 1. Artigos por tipo de estudo.

A partir dos dados coletados, verificou-se que o campo da aprendizagem móvel sofreu um aumento no volume de publicações nos periódicos indexados a SCOPUS de 2016 para 2017. Em 2016 foram 43 publicações e em 2017 foram 45 publicações. Verificou-se que o maior número de pesquisas fora realizado no ensino superior. Ao contrário dos trabalhos de Hwang \& Tsai (2011), Liu et al. (2014) e Sung et al. (2016) que relataram que o ensino fundamental foi o mais representado em suas pesquisas sobre aprendizagem móvel. Na presente pesquisa foram encontrados 4 trabalhos realizados no ensino fundamental, 3 no ensino médio, 1 na educação especial e 21 no ensino superior. Quanto ao método de pesquisa foram encontrados 9 estudos de casos, 8 questionários, 8 experimentos, 2 observações, 9 entrevistas, 2 métodos mistos e 4 vídeos. Foi encontrado somente um trabalho em ambiente informal e não foram encontrados estudos em contextos não formais, sendo a grande maioria realizados em ambientes formais. Assim como no trabalho de Wu et al. (2012) também foi relatado que os telefones móveis foram os dispositivos mais utilizados nas pesquisas realizadas.

\section{Considerações finais}

Constatou-se que o tema aprendizagem móvel, apresentou um pequeno crescimento no volume de publicações do ano de 2016 para 2017, mostrando a aparentemente continuidade do interesse por parte dos pesquisadores em aprofundar as pesquisas nessa 
área. A revista International Journal of Interactive Mobile Technologies foi a revista que mais publicou sobre o tema nos anos analisados; alguns autores se destacaram com duas publicações; a maioria dos estudos não foram identificados como qualitativa ou quantitativa, mas a maioria dos identificados são estudos qualitativos; vários tipos de métodos de pesquisa foram utilizados nos trabalhos; o ensino superior foi o cenário mais estudado nos últimos anos; a maioria dos estudos ocorreram em contextos formais de aprendizagem; e o celular foi o dispositivo mais usado nas pesquisas. Os resultados podem servir de orientação para pesquisadores, educadores ou criadores de políticas, para futuras pesquisas. As descobertas empíricas, possibilitam a discussão pautada em elementos concretos, que já foram estudados, analisados e estruturados, apresentando as lacunas a serem aprofundadas e possibilitando oportunidades de pesquisa.

\section{Referências}

Ally, M. and Prieto-Blázquez, J. (2014). What is the future of mobile learning in education? [Special Section] Revista de Universidad y Sociedad del Conocimiento (RUSC) Mobile learning applications in higher education, v. 11 (1), p. 142-151.

Crompton, H. (2013). A historical overview of mobile learning: Toward learner-centered education. Z. L. Berge, L.Y. Muilenburg (Eds.), Handbook of mobile learning, Routledge, Florence, p. 3-14.

Crompton, H.; Burke, D.; Gregory, K. and Gräbe, C. (2016). The use of mobile learning in science education: A systematic review. Journal of Science Education and Technology, v. 25, p. 149-160.

Foti, M. (2014). Mobile learning: How students use mobile devices to support learning. Journal of Literacy and Technology, v. 15 (3), p. 58-78.

Frohberg, D.; Göth, C. and Schwabe, G. (2009). Mobile learning projects - a critical analysis of the state of the art. Journal of Computer Assisted Learning, v. 25 (4), p. 307-331.

Gough, D.; Oliver, S. and Thomas, J. (Eds.) (2012). An introduction to systematic reviews, SAGE Publications, London, p. vii-x.

Hwang, G. and Tsai, C. (2011). Research trends in mobile and ubiquitous learning: A review of publication in selected journals from 2001 to 2010. British Journal of Educational Technology, v. 42 (4), p. E65-E70.

Liu, M.; Scordino, R.; Geurtz, R.; Navarrete, C.; Ko, Y. and Lim, M. (2014). A look at research on mobile learning in K-12 education from 2007 to the presente. Journal of Research on Technology in Education, v. 46 (4), p. 325-372.

Martins, E. R.; Geraldes, W. B.; Afonseca, U. R. and Gouveia, L. M. B. (2018). Tecnologias Móveis em Contexto Educativo: uma Revisão Sistemática da Literatura. RENOTE. Revista Novas Tecnologias Na Educação, v. 16, p. 1-10.

Sung, Y.; Chang, K. and Liu, T. (2016). The effects of integrating mobile devices with teaching and learning on students' learning performance: A meta-analysis and research synthesis. Computers \& Education, v. 94, p. 252-275.

Transfield, D.; Denyer, D. and Smart, P. (2003). Towards a methodology for developing evidence-informed management knowledge by means of systematic review. British Academy of Management, v. 14. p. 207-222.

Wu, W.; Wu, Y.; Chen, C.; Kao, H.; Lin, C. and Huang, S. (2012). Review of trends from mobile learning studies: A meta-analysis. Computers \& Education, v. 59 (2), p. 817-827. 